\title{
Molecular grouping of Listeria monocytogenes based on the sequence of the inlB gene
}

\author{
HENRIK ERICSSON, HELLE UNNERSTAD, JENS G. MATTSSON*, MARIE-LOUISE DANIELSSON- \\ THAM and WILHELM THAM
}

Department of Food Hygiene, Faculty of Veterinary Medicine, Swedish University of Agricultural Sciences, PO Box 7009, 75007 Uppsala and *Department of Parasitology, National Veterinary Institute, PO Box 7073, 75007 Uppsala, Sweden

The major part of the gene inlB was sequenced in 24 strains of $L$ isteria monocytogenes belonging to serovars $1 / 2 \mathrm{a}, 1 / 2 \mathrm{~b}, 1 / 2 \mathrm{c}, 3 \mathrm{~b}$ and $4 \mathrm{~b}$. A phylogenetic analysis based on the in $/ B$ nucleotide sequences showed that strains of serovars $1 / 2 a$ and $1 / 2 c$ were closely related, as well as those of serovars $1 / 2 b$ and $3 b$. Strains sharing serovar $4 b$ could be divided into two distinct groups. There were differences in amino-acid sequence between all serovars except between serovars $1 / 2 b$ and $3 b$. Differences in amino-acid sequence were also seen within each of the serovars $1 / 2 \mathrm{a}$ and $4 \mathrm{~b}$. The data presented indicate that the inlB gene may be useful for typing purposes as an alternative or complement to serotyping.

\section{Introduction}

Listeria monocytogenes is the causative organism of listeriosis, which primarily affects immunocompromised individuals, including pregnant women. Listeriosis is manifested mainly as meningitis in adult patients and as premature labour or abortion in pregnant women [1].

$\mathrm{L}$. monocytogenes is considered to have an oral route of infection, i.e., the patients who fall ill with listeriosis have ingested food that is contaminated by the bacterium [2].

The uptake of $\mathrm{L}$. monocytogenes from the gastrointestinal tract is induced by the bacterium itself by the protein internalin, which is encoded by the gene inlA [3]. Internalin induces uptake of the bacterium by normally non-phagocytic cells, e.g., the epithelial cells in the gastrointestinal mucosa. From the epithelial cells, the bacteria spread into adjacent cells and further into the bloodstream. A $n$ important site for the replication of L. monocytogenes is the liver [4]. The product of the gene inlB is essential for the bacteria to enter the hepatocytes [5]. This gene is situated downstream from inlA and belongs to the same gene family [3].

Received 2 March 1999; revised manuscript received 20 June 1999; accepted 30 June 1999.

Corresponding author: $\mathrm{Dr} \mathrm{H}$. Ericsson (e-mail: Henrik. Ericsson@Imhyg.slu.se).
A n earlier study characterised a gene segment containing the downstream part of inlA and most of inl $B$ by $P C R$ combined with restriction enzyme analysis (PCR REA) [6]. It was found that L. monocytogenes serovar 4b strains could be divided into two groups according to the PCR-REA pattern: $4 b-I$ and $4 b-I I$. Poyart et al. [7] showed that the $3^{\prime}$ end of inlA exhibits a low degree of polymorphism. Thus, it is likely that the observed differences between the two groups in the PCR-REA study emanate from inlB. To test this hypothesis, the present study sequenced the major part of the inlB gene from serovar $4 b$ strains belonging to the two PCR-REA groups. The same segment of inlB from strains belonging to serovars $1 / 2 a, 1 / 2 b, 1 / 2 c$ and $3 \mathrm{~b}$, which are the serovars most commonly found, were also sequenced and a phylogenetic analysis of the sequences was made. Furthermore, the deduced aminoacid sequences from the inlB gene products of strains representing the two serovar $4 \mathrm{~b}$ PCR-REA groups, as well as other serovars, were compared. To investigate the genetic diversity, all strains were typed by restriction endonuclease analysis (REA) and pulsedfield gel electrophoresis (PFGE).

$M$ aterials and methods

Bacterial strain

The strains were chosen from different sources, countries and years of isolation to obtain heterogenous material (Table 1). 
Table 1. Serovar, source, country and year of isolation of L. monocytogenes strains for which the inlB gene was sequenced

\begin{tabular}{|c|c|c|c|c|c|}
\hline Strain no. & Serovar & Source & Country & Year & $\begin{array}{l}\text { PFGE } \\
\text { type }\end{array}$ \\
\hline 77 & $1 / 2 a$ & Human & Sweden & 1988 & $A$ \\
\hline 83 & $1 / 2 a$ & Goat faeces & Sweden & 1987 & B \\
\hline 125 & $1 / 2 a$ & Human & Sweden & 1988 & C-1 \\
\hline 465 (АТCC 19111) & $1 / 2 a$ & Guinea-pig & England & 1926 & $C-2$ \\
\hline 1065 & $1 / 2 \mathrm{~b}$ & Human & Sweden & 1980 & E-1 \\
\hline 1419 & $1 / 2 \mathrm{~b}$ & Human & Sweden & 1958 & $\mathrm{E}-2$ \\
\hline 2324 & $1 / 2 \mathrm{~b}$ & Cheese & Canada & 1989 & $E-3$ \\
\hline 2393 & $1 / 2 b$ & Human & Sweden & 1992 & $\mathrm{~F}-1$ \\
\hline 524 & $1 / 2 \mathrm{c}$ & Human & Sweden & 1989 & D-1 \\
\hline 525 & $1 / 2 \mathrm{C}$ & Human & England & 1988 & D-1 \\
\hline 1929 & $1 / 2 \mathrm{c}$ & M edwurst & Sweden & 1993 & $D-2$ \\
\hline 2551 & $1 / 2 \mathrm{c}$ & Cheese & Denmark & 1997 & $D-2$ \\
\hline 594 & $3 b$ & Human & Germany & NK & $\mathrm{F}-2$ \\
\hline 1181 & $3 b$ & Salami & USA & 1989 & $E-3$ \\
\hline 1513 & $3 b$ & M ackerel & Sweden & 1993 & E-4 \\
\hline 1805 & $3 b$ & Trout & Sweden & 1994 & $E-3$ \\
\hline 122 & $4 b-1$ & Human & Sweden & 1988 & $\mathrm{G}-1$ \\
\hline 123 & $4 b-1$ & Human & Sweden & 1988 & $\mathrm{G}-1$ \\
\hline 501 & $4 b-1$ & Human & Sweden & 1976 & G-1 \\
\hline 520 & $4 b-1$ & Human & Sweden & 1989 & $G-2$ \\
\hline 498 & $4 b-11$ & Human & Sweden & 1974 & $\mathrm{H}-1$ \\
\hline 502 & $4 b-11$ & Human & Sweden & 1977 & $\mathrm{H}-1$ \\
\hline 526 & $4 b-11$ & Human & Sweden & 1990 & $\mathrm{H}-2$ \\
\hline 559 (NCTC 11994) & $4 b-11$ & Cheese & England & 1986 & $\mathrm{H}-1$ \\
\hline
\end{tabular}

\section{$P F G E$}

REA by PFGE was performed according to the procedure described by Unnerstad et al. [8], with the following running conditions: initial pulse time $4.0 \mathrm{~s}$ interpolated up to $40 \mathrm{~s}$ over $24 \mathrm{~h}$, with a voltage of $6 \mathrm{~V} / \mathrm{cm}$. The enzyme used was Ascl.

\section{PCR}

One colony of each strain was inoculated into $20 \mathrm{ml}$ of brain-heart infusion broth. After incubation at $37^{\circ} \mathrm{C}$ for $24 \mathrm{~h}, 15 \mu \mathrm{l}$ of each culture were mixed with $140 \mu \mathrm{l}$ of sterile water and denatured with $14 \mu \mathrm{l}$ of $0.8 \mathrm{M} \mathrm{NaOH}$ in an Eppendorf tube. The tubes were heated at $70^{\circ} \mathrm{C}$ for $10 \mathrm{~min}$, cooled on ice, and the sample was neutralised with $18 \mu \mathrm{l}$ of $1 \mathrm{M}$ Tris (pH 8.0) and $12 \mu \mathrm{l}$ of $0.8 \mathrm{M} \mathrm{HCl}$. The $\mathrm{pH}$ of the suspension was checked and considered acceptable if it was between 7 and 9 . Five $\mu \mathrm{l}$ (c. 100000 bacteria) were used for PCR in combination with the primers Lmp 23 (5' ATTAGCTGCTTTCGTCCAACCAATGAAAG 3', positions 5893-5865) and Lmp 24 (5' ACAACTGGCGATAGCGATAATGCGC 3', positions 3836-3860). The sequence data used for construction of PCR primers and the position numbers were those published previously by Gaillard et al. [3]. The PCR was performed essentially as described by Saiki et al. [9]. The PCR mixture $(50 \mu \mathrm{l})$ contained $30 \mathrm{~mm}$ Tricine, pH 8.4 (Sigma), $2.0 \mathrm{~mm} \mathrm{M} \mathrm{gCl}_{2}$, Thesit (Sigma) $0.1 \%$, $200 \mu \mathrm{M}$ of each deoxynucleoside triphosphate (dATP, dTTP, dCTP, dGTP; B oehringer M annheim), $0.2 \mu \mathrm{M}$ of each primer and 1.0 unit of AmpliTaq DNA polymerase (Perkin Elmer). PCR was performed in a Perkin Elmer thermocycler (P13480) and run for 40 cycles $\left(94^{\circ} \mathrm{C}\right.$ for $1.25 \mathrm{~min} ; 57^{\circ} \mathrm{C}$ for $1.25 \mathrm{~min} ; 72^{\circ} \mathrm{C}$ for $2 \mathrm{~min})$.

A mplified samples $(5 \mu \mathrm{l})$ were analysed by electrophoresis in agarose $2 \%$ gel and the PCR products were visualised by ethidium bromide staining $(1.5 \mu \mathrm{g} / \mathrm{ml})$ for $15 \mathrm{~min}$.

\section{Sequencing}

Nucleotide sequences were determined with an $A B$ I PRISM 377 DNA sequencer. The primers used for sequencing are listed in Table 2 . The primers were designed from a previously published sequence [3] as well as from data obtained in this study.

The sequence data were analysed with the aid of the CAP Sequence Assembly Machine (http://gcg.tigem. it/ASSEMBLY), Clustal V and DNAid. Phylogenetic analysis was conducted with the phylogenetic inference package (PHY LIP).

\section{Results}

The results of the REA-PFGE analyses are shown in Table 1. According to their restriction profile, strains were classified with capital letters. Strains with a different capital letter differed in more than three restriction fragments, corresponding to more than a single genetic event [10]. If strains differed in three or fewer restriction fragments, corresponding to a single genetic event [10], they were designated, e.g., D-1 and D-2. 
Table 2. Primers used for sequencing of inlB in $L$. monocytogenes

\begin{tabular}{llll}
\hline Primer & Nucleotide sequence $\left(5^{\prime}-3^{\prime}\right)$ & Position & Reference \\
\hline Lmp 27 & GACCTTCGATGGTTGCTTGA & $5834-5815$ & {$[3]$} \\
Lmp 28 & CTCGCACCGCTGTAAAGCTC & $5302-5321$ & {$[3]$} \\
L mp 29 & CCATTCGGGCTTCTCTATC & $5349-5330$ & {$[3]$} \\
L mp 30 & GGTCGTTCCGAATACAGTGA & $4777-4796$ & {$[3]$} \\
L mp 31 & CATAATCGCCATCATCACTT & $4853-4834$ & {$[3]$} \\
L mp 32 & GACCTAAGTTCGCTCAAGGA & $4418-4437$ & {$[3]$} \\
L mp 33 & CCAAACTGCATTCCTGGCGC & $5470-5451$ & This study \\
L mp 34 & GTAGAGGCAGGGACGCGAAT & $5029-5048$ & This study \\
L mp 36 & CGTCCAACCAATGAAAGTGG & $5862-5881$ & This study \\
L mp 37 & GCGCCAGGGAATGCAGTTTGG & $5470-5489$ & This study \\
\hline
\end{tabular}

For each strain, the sequence of c. $1500 \mathrm{bp}$ of the inlB gene was determined. The sequences are deposited in Genebank, accession nos. AF 121024-AF121047. A short segment is shown in Table 3. The DNA sequence similarity was highest $(99.8-100 \%)$ among strains within serovars $1 / 2 \mathrm{~b}, 1 / 2 \mathrm{c}$ and $3 \mathrm{~b}$. The similarity among strains within serovar $1 / 2$ a was $99.5-99.9 \%$ and within serovar $4 \mathrm{~b}$ it was $98.3-100 \%$. Sequence similarities between strains of different serovars varied from $89.2 \%$ (serovars $1 / 2 a-4 b$ ) to $100 \%$ (serovars $1 / 2 b-3 b)$. The sequencing primers initially designed functioned well when used on PCR fragments from serovars $1 / 2 a, 1 / 2 b, 1 / 2 c$ and $3 b$ strains. However, when the sequencing primers $L \mathrm{mp}$ 27-30 were used with $P C R$ products from strains of serovar $4 b$, most sequencing reactions yielded no data. The number of mismatches ranged from 1 to 6 bases between primers L mp 27 and 30 and the serovar 4b strains. Therefore, these primers were replaced by a set of newly designed primers, Lmp 33, Lmp 34, L mp 36 and Lmp 37. The polymorphism in the inlB gene of the various strains

Table 3. Partial nucleotide sequences of inlB in different serovars of $L$. monocytogenes

\begin{tabular}{|c|c|c|}
\hline Strain no. & Serovar & Nucleotide sequence \\
\hline 77 & $1 / 2 a$ & САATTACTACATGGTATCAAT \\
\hline 83 & $1 / 2 a$ & СААТTACTACATGGTATCAAT \\
\hline 125 & $1 / 2 a$ & СААТTAСTACATGGTATCAAT \\
\hline 465 & $1 / 2 a$ & СААТTAСТАСАTGGTATСААТ \\
\hline 1065 & $1 / 2 b$ & СААТTАСТАСТTGGTАTСААТ \\
\hline 1419 & $1 / 2 b$ & СААТTАСТАСТTGGTАТСААТ \\
\hline 2324 & $1 / 2 b$ & САATTACTACTTGGTATCAAT \\
\hline 2393 & $1 / 2 b$ & СААТTАСТАСТTGGTАТСААТ \\
\hline 524 & $1 / 2 \mathrm{C}$ & СААТTАСТАСАTGGTАTСААТ \\
\hline 525 & $1 / 2 \mathrm{C}$ & СААТTAСТАСАTGGTATCAAT \\
\hline 1929 & $1 / 2 c$ & СААТTAСТАСАTGGTATСААТ \\
\hline 2551 & $1 / 2 c$ & СААТTAСТАСАTGGTATСААТ \\
\hline 594 & $3 b$ & СААТTAСTAСTTGGTATCAAT \\
\hline 1181 & $3 b$ & СААТTАСТАСТTGGTATСААТ \\
\hline 1513 & $3 b$ & СААТTAСТАСТTGGTATСААТ \\
\hline 1805 & $3 b$ & САATTAСTACTTGGTATCAAT \\
\hline 122 & $4 b-I$ & СAATTACCACGTGGTATCAAT \\
\hline 123 & $4 b-I$ & СААTTACCACGTGGTATCAАT \\
\hline 501 & $4 b-1$ & CAATTACCACGTGGTATCAAT \\
\hline 520 & $4 b-1$ & САATTACCACGTGGTATCAAT \\
\hline 498 & $4 b-11$ & САATTACTACGTGGTATCAAT \\
\hline 502 & $4 b-11$ & СААТTACTACGTGGTATCAАT \\
\hline 526 & $4 b-11$ & CAATTACTACGTGGTATCAAT \\
\hline 559 & $4 b-I I$ & $\begin{array}{l}\text { CAATTACTACGTGGTATCAAT } \\
\star * * * * * * * * * * * * * * * * * *\end{array}$ \\
\hline Position & & 5586 \\
\hline
\end{tabular}

was converted into a phylogenetic tree (Fig. 1) by a comparative analysis. In this analysis, the strains formed clusters in agreement with their serovars. A comparison of the deduced amino-acid sequences demonstrated that amino-acid substitutions were present between all serovars except between serovars $1 / 2 b$ and $3 \mathrm{~b}$. There were also amino-acid substitutions between inlB genes within each of the serovars $1 / 2 a$ and $4 \mathrm{~b}$ (Table 4). The amino-acid sequence similarities between serovars were between $91.9 \%$ (serovars $1 / 2 a$ and $4 b$ ) and $100 \%$ (serovars $1 / 2 b$ and $3 b$ ).

Strains within serovar $1 / 2 a$ displayed amino-acid similarities of $99.4-100 \%$ and strains within serovar 4b showed $98.6-100 \%$ similarity.

\section{Discussion}

In an earlier study, 133 L. monocytogenes strains sharing serovar $4 \mathrm{~b}$ could be divided into two groups (4b-I and 4b-II) based on a PCR-REA of the $3^{\prime}$ end of inl $A$ and nearly the entire inlB gene [6]. The present study, based on the amplification and sequencing of $C$. $1500 \mathrm{bp}$ (out of $1890 \mathrm{bp}$ ) of inlB obtained the same grouping as above, i.e., strains belonging to serovar $4 b$ could be divided into two groups, identical with $4 \mathrm{~b}-\mathrm{I}$ and $4 \mathrm{~b}-\mathrm{II}$. Thus, it seems likely that these two PCRREA groups solely reflect differences in the inlB sequences. This is also in agreement with Poyart et al. [7] who showed that the variability in the $3^{\prime}$ end of the inlA gene is very low. The inlB sequence data obtained not only support a division of strains belonging to serovar $4 b$, but also of strains of serovar $1 / 2 a$ (Fig. 1). This has also been shown recently by PCR-REA [11]. At the amino-acid level, strains of groups $4 b-I$ and $4 b-$ II differ in six amino acids, whereas there are differences in three amino acids between the strains representing the two $1 / 2$ a groups. In contrast, the strains of serovar $1 / 2 b$ and $3 b$ differ to some extent in their inlB DNA sequences, but the corresponding amino-acid sequences are identical.

The fact that primers $L \mathrm{mp} 27-30$ did not function well when used on PCR products from serovar $4 \mathrm{~b}$ strains is not surprising, as the sequence data published by Gaillard et al. [3] and upon which primers L mp 27-30 


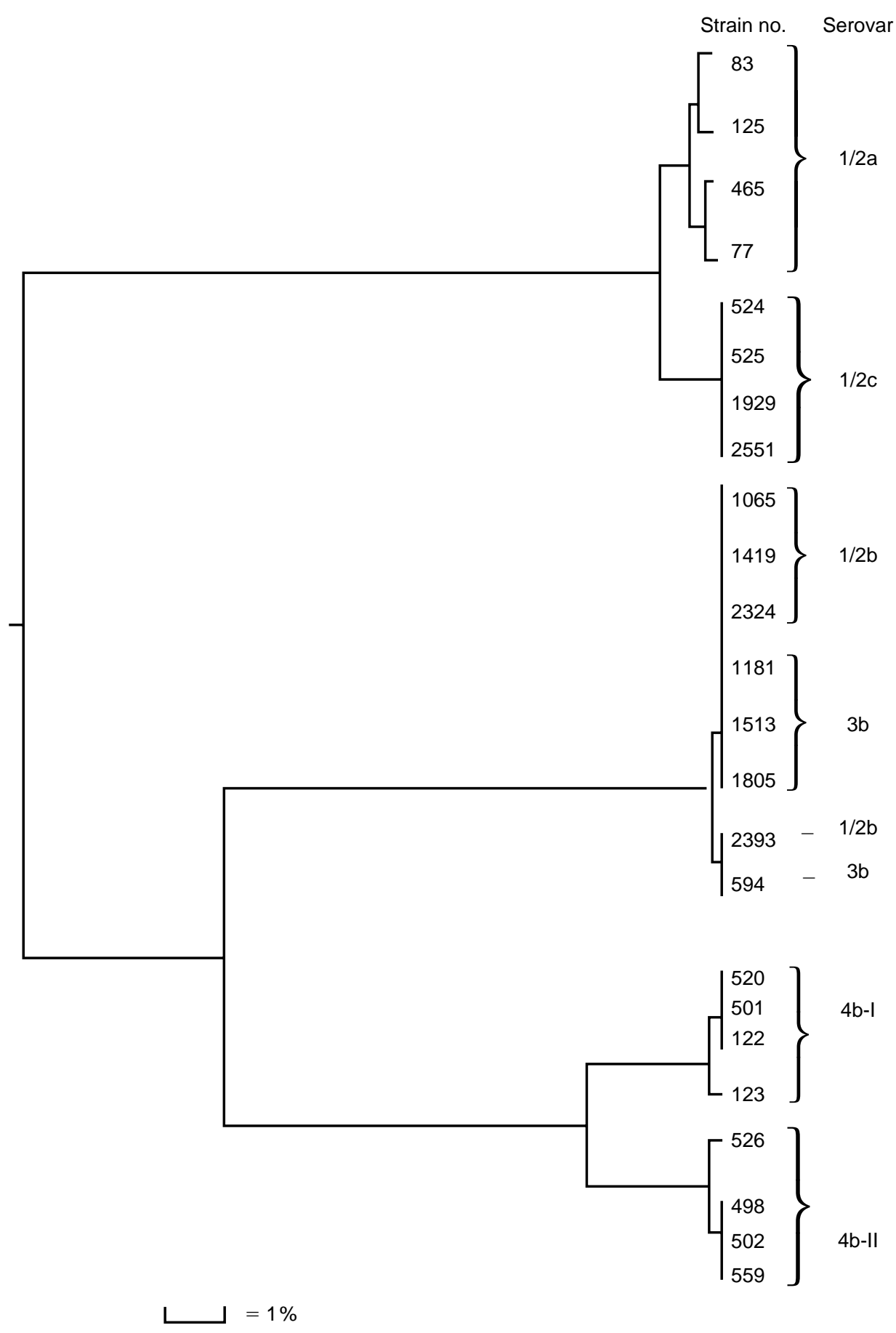

Fig. 1. The relationship between different strains of $L$. monocytogenes inferred from nucleotide sequence polymorphism of the inlB gene. The tree was assembled with the aid of the average linkage clustering method in the NEIGHBOR program of PHYLIP.

are based, emanate from a serovar $1 / 2$ a strain. The strains of serovar $4 b$ showed the lowest sequence similarity with serovar $1 / 2 a$ and, therefore, may need specially designed primers for certain genomic regions. This was also the case in the present study.

It is noteworthy that in this study, as well as in other studies where a division has been made based on polymorphism in virulence-associated genes, serovars $1 / 2 \mathrm{a}$ and $1 / 2 \mathrm{c}$ cluster together $[12,13]$. In the study by Vines et al. [13], strains of serovars $1 / 2 b, 3 b$ and $4 b$ were also grouped together, whereas in the present study the members of serovar $4 b$ formed their own group, but from the same branch as the $1 / 2 b-3 b$ strains. Serotyping, based on $O$ and $H$ antigens, is probably the oldest and most established typing method for L. monocytogenes. It is an interesting observation that there seems to be a correlation between the serovars and the structure of virulence-associated genes. This indicates that the evolution of the genes encoding the $\mathrm{O}$ and $\mathrm{H}$ antigens has been parallel to the evolution of virulence-associated genes. This has also 
Table 4. Deduced amino-acid sequences from the major part of inlB in different serovars of $L$. monocytogenes

\begin{tabular}{|c|c|c|}
\hline Strain no. & Serovar & A mino-acid sequence \\
\hline $\begin{array}{l}77,465 \\
83 \\
125 \\
1065,1419 \\
2324,2393 \\
594,1181 \\
1513,1805 \\
524,525 \\
1929,2551 \\
122,501 \\
520 \\
123 \\
498,502 \\
526,559\end{array}$ & $\begin{array}{l}1 / 2 a \\
1 / 2 a \\
1 / 2 a \\
1 / 2 b \\
1 / 2 b \\
3 b \\
3 b \\
1 / 2 c \\
1 / 2 c \\
4 b-1 \\
4 b-1 \\
4 b-1 \\
4 b-11 \\
4 b-11\end{array}$ & 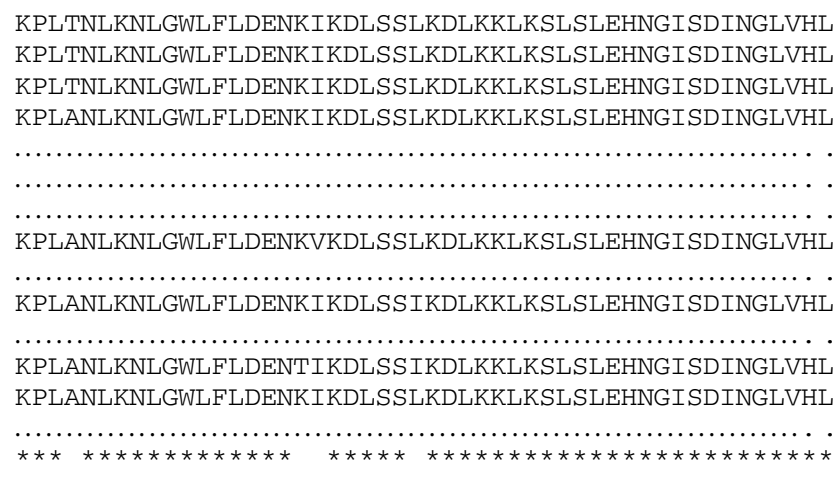 \\
\hline
\end{tabular}

$\begin{array}{ll}77,465 & 1 / 2 \\ 83 & 1 / 2 a \\ 125 & 1 / 2 a \\ 1065,1419, & 1 / 2 b \\ 2324,2393, & 1 / 2 b \\ 594,1181, & 3 b \\ 1513,1805 & 3 b \\ 524,525, & 1 / 2 \\ 1929,2551 & 1 / 2 \\ 122,501, & 4 b- \\ 520 & 4 b- \\ 123 & 4 b- \\ 498,502, & 4 b- \\ 526,559 & 4 b-\end{array}$

$\begin{array}{ll}77,465 & 1 / 2 a \\ 83 & 1 / 2 a \\ 125 & 1 / 2 a \\ 1065,1419, & 1 / 2 b \\ 2324,2393, & 1 / 2 b \\ 594,1181, & 3 b \\ 1513,1805 & 3 b \\ 524,525, & 1 / 2 c \\ 1929,2551 & 1 / 2 c \\ 122,501, & 4 b-1 \\ 520 & 4 b-1 \\ 123 & 4 b-1 \\ 498,502, & 4 b-11 \\ 526,559 & 4 b-11\end{array}$

PQLESLYLGNNKITDITVLSRLTKLDTLSLEDNQISDIVPLAGLTKLQNL PQLESLYLGNNKITDITVLSRLTKLDTLSLEDNQISDIVPLAGLTKLQNL PQLESLYLGNNKITDITVLSRLTKLDTLSLEDNQISDIVPLAGLTKLQNL LQLESLYLGNNKLTDITILSRLTKLDTLSLEDNEISDIVPLSGLTKLQNL

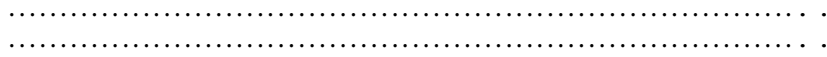
PQLESLYLGNNKITDITVLSRLTKLDTLSLEDNQISDIVPLAGLTKLQNL

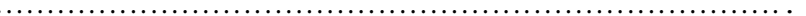
PQLESLYLGNNKLTDITILSRLTKLDTLSLEDNEISDIVPLSGLTKLQNL PQLESTYLGNNKLTDITITSRITKLDTISL EDNETSDIVPISGLTKLQNL LQLESLYLGNNKLTDITILSRLTKLDTLSLEDNEISDIVPLSGLTKLQNL

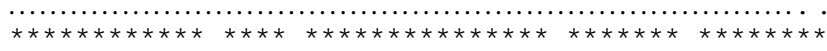

YLSKNHISDLRALAGLKNLDVLELFSQECLNKPINHQSNLVVPNTVKNTD YLSKNHISDLRALAGLKNLDVLELFSQECLNKPINHQSNLVVPNTVKNTD YLSKNHISDLRALAGLKNLDVLELFSQECLNKPINHQSNLVVPNTVKNTD YLSKNHISDLRALAGLKNLDVLELFSQECLNKSINHQTNLVVPNTVKNID

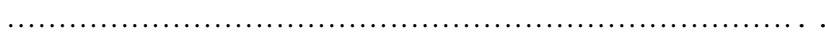

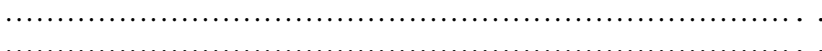
YLSKNHISDLRALAGLKNLDVLELFSQECLNKPINHQSNLVVPNTVKNTD YLSKNHISDLRALAGLKNLDVLELFSQECLNKSINHQTNLVVPNTVKNID

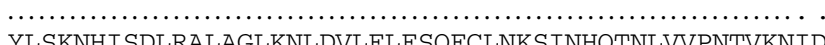
YLSKNHISDLRALAGLKNLDVLELFSQECLNKS INHQMNLVVPNTVKNID

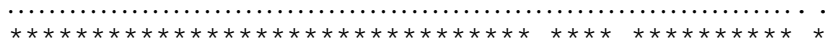

$\begin{array}{ll}77,465 & 1 / 2 a \\ 83 & 1 / 2 a \\ 125 & 1 / 2 a \\ 1065,1419, & 1 / 2 b \\ 2324,2393, & 1 / 2 b \\ 594,1181, & 3 b \\ 1513,1805 & 3 b \\ 524,525, & 1 / 2 c \\ 1929,2551 & 1 / 2 c \\ 122,501, & 4 b-1 \\ 520 & 4 b-1 \\ 123 & 4 b-1 \\ 498,502, & 4 b-11 \\ 526,559 & 4 b-11 \\ & \\ 77,465 & 1 / 2 a \\ 83 & 1 / 2 a \\ 125 & 1 / 2 a \\ 1065,1419, & 1 / 2 b \\ 2324,2393, & 1 / 2 b \\ 594,1181, & 3 b \\ 1513,1805 & 3 b \\ 524,525, & 1 / 2 c \\ 1929,2551 & 1 / 2 c \\ 122,501, & 4 b-1 \\ 520 & 4 b-1 \\ 123 & 4 b-1\end{array}$

GSLVTPEIISDDGDYEKPNVKWHLPEFTNEVSFIFYQPVTIGKAKARFHG GSLVTPEIISDDGDYEKPNVKWHLPEFTNEVSFIFYQPVTIGKAKARFHG GSLVTPEIISDDGDYEKPNVKWHLPEFTNEVSFIFYQPVTIGKAKARFHG GSLVTPEIISDDGDYEKPNVKWHLPEF INEVSF IFYQPVTVGKAKARFHG

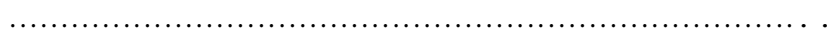
GSLVTPEIISDDGDYEKPNVKWHLPEFTNEVSFIFYQPVTIGKAKARFHG $\ldots \ldots \ldots \ldots \ldots \ldots \ldots \ldots \ldots \ldots \ldots \ldots \ldots \ldots \ldots \ldots \ldots \ldots \ldots \ldots \ldots \ldots \ldots \ldots \ldots \ldots \ldots \ldots \ldots$
GSLVTPEIISDDGDYEKPNVKWHLPEF INEVSFIFYQPVTVGKAKARFHG GSLVTPEIISDDGDYEKPNVKWHLPEF INEVSFIFYQPVTVGKAKARFHG GSLVTPEIISDDGDYEKPNVKWHLPEF INEVSF IFYQPVTVGKAKARFHG

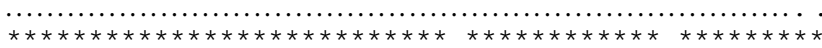
RVTQPLKEVYTVSYDVDGTVIKTKVEAGTRITAPKPPTKQGYVFKGWYTE RVTQPLKEVYTVSYDVDGTVIKTKVEAGTRITAPKPPTKQGYVFKGWYTE RVTQPLKEVYTVSYDVDGTVIKTKVEAGTRITAPKPPTKQGYVFKGWYTE RVTQPLKEVYTVSYDVDGTVIKTKVEAGTRITAPKPPTKQGYVFKGWYTE

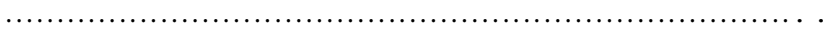

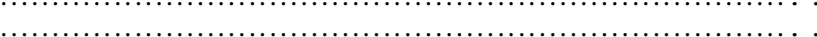
RVTQPLKEVYTVSYDVDGTVIKTKVEAGTRITAPKPPTKQGYVFKGWYTE

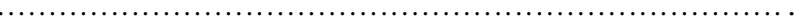
RVTQPLKEVYTVSYDVDGTVIKTKVEAGTRITAPKPPTKQGYVFKGWYTE RVTQPLKEVYTVSYDVDGTVIKTKVEAGTRITAPKPPTKQGYVFKGWYTE 
Table 4. (continued)

\begin{tabular}{|c|c|c|}
\hline Strain no. & Serovar & A mino-acid sequence \\
\hline $\begin{array}{l}498,502 \\
526,559\end{array}$ & $\begin{array}{l}4 b-11 \\
4 b-11\end{array}$ & 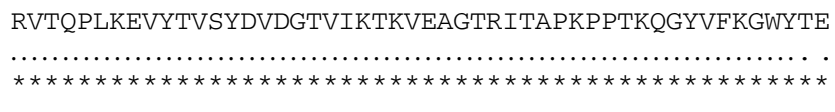 \\
\hline
\end{tabular}

$\begin{array}{ll}77,465 & 1 / 2 a \\ 83 & 1 / 2 a \\ 125 & 1 / 2 a \\ 1065,1419, & 1 / 2 b \\ 2324,2393, & 1 / 2 b \\ 594,1181, & 3 b \\ 1513,1805 & 3 b \\ 524,525, & 1 / 2 c \\ 1929,2551 & 1 / 2 c \\ 122,501, & 4 b-1 \\ 520 & 4 b-1 \\ 123 & 4 b-1 \\ 498,502, & 4 b-1 \\ 526,559 & 4 b-1\end{array}$

KNGGHEWNFNTDYMSGNDFTLYAVFKAETTEKAVNLTRYVKYIRGNAGIY KNGGHEWNFNTDYMSGNDFTLYAVFKAETTEKAVNLTRYVKYIRGNAGIY KNGGHEWNFNTDYMSGNDFTLYAVFKAETTEKAVNLTRYVKYIRGNAGIY KNGGHEWDFSTDYMSGNDFTLYAMFKVETTEKAVNLTRYVKYIRGNAGIY

$1 / 2 b$

$1 / 2 b$

$3 b$

$3 \mathrm{~b}$

$1 / 2 \mathrm{c}$

$4 b-1$

$4 b-1$

$4 b-1$

$4 b-11$

$\begin{array}{ll}77,465 & 1 / 2 a \\ 83 & 1 / 2 a \\ 125 & 1 / 2 a \\ 1065,1419, & 1 / 2 b \\ 2324,2393, & 1 / 2 b \\ 594,1181, & 3 b \\ 1513,1805 & 3 b \\ 524,525, & 1 / 2 c \\ 1929,2551 & 1 / 2 c \\ 122,501, & 4 b--1 \\ 520 & 4 b-1 \\ 123 & 4 b-1 \\ 498,502, & 4 b-11 \\ 526,559 & 4 b-11\end{array}$

$77,465 \quad 1 / 2 a$

$83 \quad 1 / 2 a$

$125 \quad 1 / 2 a$

$1065,1419, \quad 1 / 2 b$

2324, 2393, $\quad 1 / 2 b$

$594,1181, \quad 3 b$

$524,525, \quad 1 / 2 \mathrm{C}$

$1929,2551 \quad 1 / 2 \mathrm{C}$

122, 501, 4b-I

5204 b-

$123 \quad 4 \mathrm{~b}-\mathrm{-}$

$498,502, \quad 4 b-11$

526, 5594 4b-II

$77,465 \quad 1 / 2 a$

$83 \quad 1 / 2 a$

$125 \quad 1 / 2 a$

$1065,1419, \quad 1 / 2 b$

2324, 2393, $\quad 1 / 2 b$

594, 1181, 3b

1513, $1805 \quad 3 \mathrm{~b}$

$524,525, \quad 1 / 2 \mathrm{C}$

1929, $2551 \quad 1 / 2 \mathrm{c}$

$122,501, \quad 4 b-1$

$5204 \mathrm{~b}-\mathrm{I}$

$123 \quad 4 b-1$

$498,502, \quad 4 b-11$

526, 5594 4b-II

$77,465 \quad 1 / 2 a$

$83 \quad 1 / 2 a$

$125 \quad 1 / 2 a$

$1065,1419, \quad 1 / 2 b$

2324, 2393, $\quad 1 / 2 b$

594, 1181, 3b

$\begin{array}{ll}1513,1805 & 3 \mathrm{~b} \\ 524,525 & 1 / 2 \mathrm{c}\end{array}$

$1929,2551 \quad 1 / 2 \mathrm{C}$

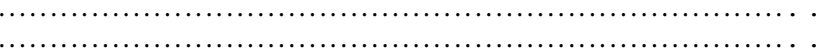

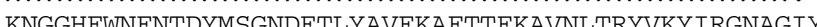

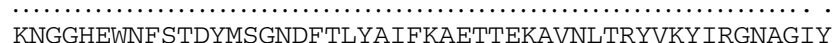
KNGGHEWNFSTDYMSGNDFTLYAIFKAETTEKAVNLTRYVKYIRGNAGIY KNGGHEWNFSTDYMSGNDFTLYAMFKAETTEKAVNLTRYVKYIRGNAGIY

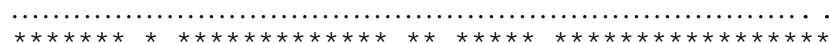

KLPREDNSLKQGTLASHRCKALTVDREARNGGKLWYRLKNIGWTKAENLS KLPREDNSLKQGTLASHRCKALTVDREARNGGKLWYRLKNIGWTKAENLS KLPREDNSLKQGTLASHRCKALTVDREARNGGKLWYRLKNIGWTKAENLS KLPREDNSLKQGTLASHRCKALTVDREARNGGKLWYRLKNIGWTKAENLS

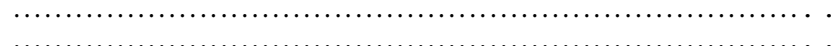
KLPREDNSLKQGTLASHRCKALTVDREARNGSKLWYRLKNIGWTKAENLS $\ldots \ldots \ldots \ldots \ldots \ldots \ldots \ldots \ldots \ldots \ldots \ldots \ldots \ldots \ldots \ldots \ldots \ldots \ldots \ldots \ldots$
KLPREDNSLKQGTLASHRCKALTVDREARNGSELWYRLKNIGWTKAENLS KLPREDNSLKQGTLASHRCKALTVDREARNGSELWYRLKNIGWTKAENLS KLPREDNSLKQGTLASHRCKALTVDREARNGGELWYRLKNIGWTKAENLS

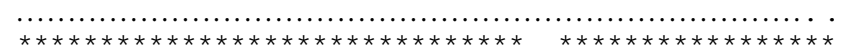

LDRYDKMEYDKGVTAYARVRNASGNSVWTKPYNTAGAKHVNKLSVYQGKN LDRYDKMEYDKGVTAYARVRNASGNSVWTKPYNTAGAKHVNKLSVYQGKN LDRYDKMEYDKGVTAYARVRNASGNSVWTKPYNTAGAKHVNKLSVYQGKN LDRYDKIEYDKGVTAYARVKNALGNAVWTKPYNTAGAKHVNKLSVYQGKN

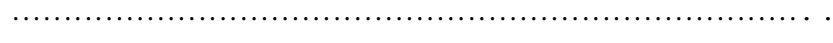

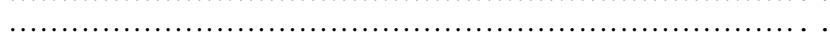
LDRYDKMEYDKGVTAYARVRNASGNSVWTKPYNTAGAKHVNKLSVYQGKN LDRYDKIEYDKGVTAYARVKNAPGNAVWTKPYNTAGATLVNKLSVYQGKN

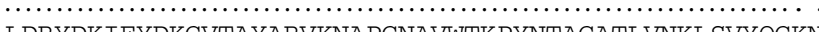
LDRYDKIEYDKGVTAYARVKNAPGNAVWTKPYNTAGATLVNKLSVYQGKN

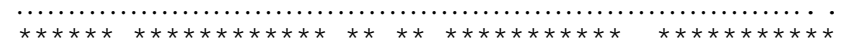

MRILREAKTPITTWYQFSIGGKVIGWVDTRALNTFYKQSMEKPTRLTRYV MRILREAKTPITTWYQFSIGGKVIGWVDTRALNTFYKQSMEKPTRLTRYV MRILREAKTPITTWYQFSIGGKVIGWVDTRALNTFYKQSMEKPTRLTRYV MRILREAKTPITTWYQFSIGGKVIGWVDTRALNTFYKQSMEKPTRLTRYV

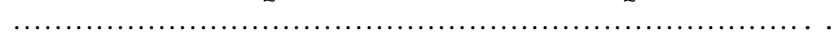

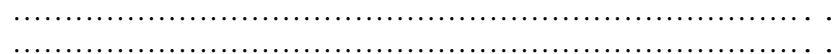
MRILREAKTPITTWYQFSIGGKVIGWVDTRALNTFYKQSMEKPTRLTRYV MRILREAKTPITTWYQFS IDGKVIGWVDTRALNTFYKQSMEIPIQLTRYV $\ldots \ldots \ldots \ldots \ldots \ldots \ldots \ldots \ldots \ldots \ldots \ldots \ldots \ldots \ldots \ldots \ldots \ldots \ldots \ldots \ldots \ldots \ldots$
MRILREAKTPITTWYQFSIDGKVIGWVDTRALNTFYKQ SMEIPIQLTRYV MRILREAKTPITTWYQFSIDGKVIGWVDTRALNTFYKQSMEIPIQLTRYV

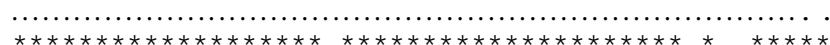

SANKAGESYYKVPVADNPVKRGTLAKYKNQKLIVDCQATIEGQLWYRIRT SANKAGESYYKVPVADNPVKRGTLAKYKNQKLIVDCQATIEGQLWYRIRT SANKAGESYYKVPVADNPVKRGTLAKYKNQKLIVDCQATIEGQPWYRIRA SASKGNEAYYKVPVADNPVKRGTLAKYKNQKLIVDCQATVEGQLWYRIRT

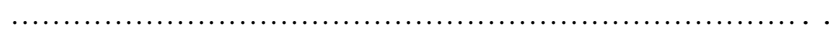

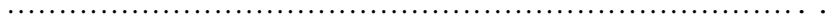
SANKAGESYYKVPVADNPVKRGTLAKYKNQKLIVDCQATIEGQLWYRIRT 
Table 4. (continued)

\begin{tabular}{|c|c|c|}
\hline Strain no. & Serovar & A mino-acid sequence \\
\hline $\begin{array}{l}122,501, \\
520 \\
123 \\
498,502, \\
526,559 \\
\\
77,465 \\
83 \\
125 \\
1065,1419, \\
2324,2393, \\
594,1181 \text {, } \\
1513,1805 \\
524,525 \\
1929,2551 \\
122,501, \\
520 \\
123 \\
498,502, \\
526,559\end{array}$ & $\begin{array}{l}4 b-1 \\
4 b-1 \\
4 b-1 \\
4 b-11 \\
4 b-11 \\
\\
1 / 2 a \\
1 / 2 a \\
1 / 2 a \\
1 / 2 b \\
1 / 2 b \\
3 b \\
3 b \\
1 / 2 c \\
1 / 2 c \\
4 b-1 \\
4 b-1 \\
4 b-1 \\
4 b-11 \\
4 b-11\end{array}$ & 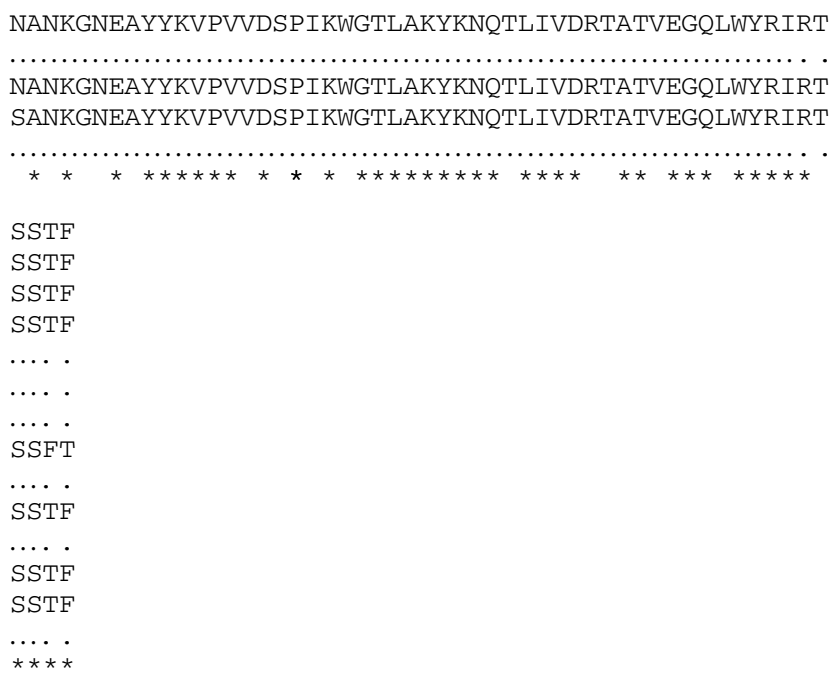 \\
\hline
\end{tabular}

*Consensus between all tested serovars; ...., same sequence as line above.

been suggested by other authors, e.g., Vines et al. [13]. Similarly, the results of the REA-PFGE analyses in the present study show that the overall genomic variability between most strains seems to correspond to the serovars. The serovars $1 / 2 b$ and $3 b$ appear to be genetically very closely related, as both the PFGE types and the inlB sequences within the strains are almost identical. This has also been reported elsewhere [14].

It should be noted that the inlB sequence of serovar 1/2a strain no. 465 (ATCC 19111) isolated in England from a guinea-pig in 1926 is almost identical to the inlB sequence of serovar $1 / 2 a$ strain no. 77 isolated from a human patient in Sweden in 1988, and that in inlB sequence of serovar 1/2b strain no. 2324 isolated in 1989 from a cheese in Canada has an identical inlB sequence to that of serovar $1 / 2 \mathrm{~b}$ strain no. 1419 isolated from a human patient in Sweden 1958. Furthermore, $>200$ L. monocytogenes strains of serovars $4 b$ and $1 / 2 a$ were analysed in the two above-mentioned PCR-REA studies $[6,11]$ and only two variants of each serovar were found. These data suggest that the inlB gene is stable within the serovars and that it could serve as a genetic marker for serovar identification.

In a paper by Vines et al. [15] three virulenceassociated genes (hlyA, plcA and inlA) in two serovar $4 \mathrm{~b}$ strains and two serovar $1 / 2 \mathrm{~b}$ strains were characterised. This set of genes from the analysed strains exhibited a higher degree of conservation than the inlB gene segment in the strains of the present study. The similarity in nucleotides, when compared with published sequences from strains of serovars $1 / 2 a$ and $1 / 2 c$, was between $97.3 \%$ and $98.8 \%$ and at the aminoacid level was between $97.8 \%$ and $99.4 \%$. In the present study, the corresponding values between these serovars were $90.7-93.4 \%$ and $92.7-95.0 \%$. This indicates that inlB may be better suited than the genes mentioned for differentiation of $L$. monocytogenes, especially between different serovars.

Based on the results of previous PCR-REA study and those of the present study, the in $B$ region may be used for typing purposes as an alternative or complement to serotyping. That could, in the near future, be done by the technique described recently by Ronaghi et al. [16]. This is a sequencing-by-synthesis method based on real-time pyrophosphate detection. The method aims to achieve rapid and automated determination of short DNA sequences. If for example, positions 5893 and 5896 (Table 3) were used, it would be possible to discriminate rapidly between four categories of $L$. monocytogenes: serovar $4 b-I$, serovar $4 b-I I$, serovars $1 / 2 b$ and $3 b$, and serovars $1 / 2 a$ and $1 / 2 c$. This could provide a useful tool for the epidemiological investigation of listeriosis.

This project was supported financially by the Swedish Council for Forestry and A gricultural Research and by the research foundation of Ivar and Elsa Sandberg, to whom we express our gratitude.

\section{Refer ences}

1. Nieman RE, Lorber B. Listeriosis in adults: a changing pattern. Report of eight cases and review of the literature, 1968-1978. Rev Infect Dis 1980; 2: 207-227.

2. James SM, Fannin SL, A gee BA et al. Listeriosis outbreak associated with Mexican-style cheese - California. Morbid Mortal Weekly Rep 1985; 34: 357-359.

3. Gaillard J-L, Berche P, Frehel C, Gouin E, Cossart P. Entry of $L$. monocytogenes into cells is mediated by internalin, a repeat protein reminiscent of surface antigens from gram-positive cocci. Cell 1991; 65: 1127-1141.

4. Gregory SH, Sagnimeni AJ, Wing EJ. Internalin B promotes the replication of Listeria monocytogenes in mouse hepatocytes. Infect Immun 1997; 65: 5137-5141.

5. Dramsi S, Biswas I, Maguin E, B raun L, Mastroeni P, Cossart P. Entry of Listeria monocytogenes into hepatocytes requires expression of InIB, a surface protein of the internalin 
multigene family. Mol Microbiol 1995; 16: 251-261.

6. Ericsson $H$, Stålhandske $P$, Danielsson-Tham $M-L$ et al. Division of Listeria monocytogenes serovar $4 \mathrm{~b}$ strains into two groups by PCR and restriction enzyme analysis. Appl Environ Microbiol 1995; 61: 3872-3874.

7. Poyart $C$, Trieu-Cuot $P, B$ erche $P$. The inlA gene required for cell invasion is conserved and specific to Listeria monocytogenes. Microbiology 1996; 142: 173- 180.

8. Unnerstad H, Bannerman E, Bille J, Danielsson-Tham M L, Waak $E$, Tham W. Prolonged contamination of a dairy with Listeria monocytogenes. Neth Milk Dairy J 1996; 50: 493- 499.

9. Saiki RK, Gelfand DH, Stoffel $S$ et al. Primer-directed enzymatic amplification of DNA with a thermostable DNA polymerase. Science 1988; 239: 487-491.

10. Tenover FC, Arbeit RD, Goering RV et al. Interpreting chromosomal DNA restriction patterns produced by pulsedfield gel electrophoresis: criteria for bacterial strain typing. J Clin Microbiol 1995; 33: 2233-2239.

11. Unnerstad $\mathrm{H}$, Nilsson I, Ericsson $\mathrm{H}$ et al. Division of Listeria monocytogenes serovar $1 / 2 a$ strains into two groups by $P C R$ and restriction enzyme analysis. Appl Environ Microbiol 1999; 65: 2054- 2056.

12. Piffaretti J-C, K ressebuch $H$, A eschbacher $M$ et al. Genetic characterization of clones of the bacterium Listeria monocytogenes causing epidemic disease. Proc Natl Acad Sci USA 1989; 86: 3818-3822.

13. Vines $A$, Reeves $M W$, Hunter $S$, Swaminathan B. Restriction fragment length polymorphism in four virulence-associated genes of Listeria monocytogenes. Res Microbiol 1992; 143: 281-294.

14. Brosch R, Chen J, Luchansky JB. Pulsed-field fingerprinting of Listeriae: identification of genomic divisions for Listeria monocytogenes and their correlation with serovar. Appl Environ Microbiol 1994; 60: 2584-2592.

15. Vines A, Swaminathan B. Identification and characterization of nucleotide sequence differences in three virulence-associated genes of Listeria monocytogenes strains representing clinically important serotypes. Curr Microbiol 1998; 36: 309-318.

16. Ronaghi $M$, Uhlén $M$, Nyrén $P$. A sequencing method based on real-time pyrophosphate. Science 1998; 281: 363-365. 